Aus der Augenklinik.

\title{
Prognose und Therapie der perforierenden infizierten Augapfelverletzungen.
}

\author{
Von Prof. Dr. 0. Schirmer.
}

Es ist eine Tatsache, der in unseren statistischen Arbeiten immer noch viel zu wenig Rechnung getragen wird, daB die Prognose der perforierenden Bulbusverletzungen fast ausschlieblich davon abhängt, ob die Wunde infiziert is t oder nicht. Ich kenne keine derartige Publikation, welche für die Stellung der Prognose die Infektionsfrage als Einteilungsprinzip benutzte; überall sind die Fälle nach dem Sitz der Wunde eingeteilt (z. B. Hillemanns ${ }^{1}$ ), Ohlemann ${ }^{2}$ ), Rö mer ${ }^{3}$ ), Silex ${ }^{4}$; wie oft eine Infektion vorlag, ist meist überhaupt nicht zu ersehen. Es fehlen uns also brauchbare Statistiken über die Prognose der perforierenden Wunden noch völlig.

Es ist deshalb anzunehmen, daß es im wesentlichen die persönlichen Erfahrungen des Autors sind, auf Grund deren in unseren Lehrbüchern die Prognose bei den infizierten Verletzungen so überaus schlecht gestellt wird. So sagt z. B. Fuchs in der 10. Auflage seines Lehrbuchs (1905):

„In den akutesten Fällen handelt es sich um eine eitrige Entzündung, welche zur Vereiterung des ganzen Auges führt - Panophthalmitis. In den weniger heftigen Fällen kommt es zur plastischen Irido-Cystitis." "Das Auge ist auch in diesem Falle meist verloren."

Und in unserem jüngsten Spezialwerk über die Verletzungen des Auges (Wiesbaden 1899) sagt Praun von den eitrigen Infektionen:

„Die Prognose ist sehr ungünstig, weil das Auge unter großen Schmerzen in der Regel rettungslos verloren geht ${ }^{\text {, }}$ und von der fibrinösen Irido-Cyclitis:

„Die Prognose ist durchaus schlecht. Ist der Prozeß einmal im Gange, so darf man froh sein, ein nicht zu kleines, nicht mehr schmerzhaftes Auge zu erhalten; die Erblindung ist immer unabwendbar."

Eine solche Vorhersage ist nach meinen eigenen Erfahrungen viel zu pessimistisch. Die Resultate, die mir eine konsequente Durchführung der gleich zu schildernden Therapie innerhalb der letzten zehn Jahre ergeben hat, berechtigen mich zu der Behauptung, daß es bei richtiger und frühzeitig eingeleiteter Therapie gelingt, etwa $z w e i$ Drittel der infizierten Augäpfel zu erhalten und davon einen recht erheblichen Prozentsatz mit brauchbarer Sehschärfe.

1) Ueber Verletzungen des Auges. Archiv fuir Augenheilkunde 1895. Bd. 30, S. 29 . - 2) ibid. 1891 , Bd. 22, S 94. - 3) Zeitschrift für praktische Aerzte 1899. 4) Beriner medizinische Wochenschrift 1888, S. 396. 
Das Studium des klinischen Bildes der sympathischen Augenentzündung, das mich längere Zeit fast ausschlieblich beschäftigt hat, gab mir die Anregung, das Quecksilber, dessen dominierende Rolle in der Therapie der sympathischen Ophthalmie allseitig anerkannt wird, auch bei den infizierten Bulbusverletzungen $\mathrm{zu}$ versuchen, bei welchen schon Albrecht v. Graefe kräftige Kalomeldosen gegeben hat. Ich hatte um so mehr Anlab, diesen Versuch zu machen, als mich meine klinischen Untersuchungen zu der damals noch nicht allgemein anerkannten Anschauung geführt hatten, daß dieselben Mikrobien, welche die sympathische Entzündung erregen, zugleich die Ursache einer Form der traumatisch-infektiösen Bulbusentzündung sind, nämlich der Uveitis fibrinosa, womit ich allerdings nicht behaupten will, daß ich diese für eine ätiologische Einheit halte. Ich übertrug dann die Behandlungsweise auch auf die Uveitis purulenta und schlieblich auch auf die bösartigste Form derselben, auf den Glaskörperabsceb. Da die direkten Infektionen gewöhnlich viel akuter einsetzen, als die sympathische Entziindung, habe ich mit den Jahren folgende etwas abweichende Behandlungsmethode herausgebildet.

Das Hauptgewicht lege ich darauf, den Körper möglichst schnell mit Quecksilberzu überschwemmen. Ich habe mich überzeugt, daß geringere Gaben nicht entfernt eine gleich günstige Wirkung haben, und daß dieselbe meist zu spät kommt. Ich lasse deshalb erwachsene Personen je nach ihrem Kräftezustande 6-9 g Ung. cinereum täglich einreiben, morgens und abends die Hälfte, kleine Kinder etwa $1 / 2-3 \mathrm{~g}$. Nur wo Inunktionen von der Haut nicht gut vertragen werden, verwende ich dafür den Mercolintschurz zu $50 \mathrm{~g}$ Ung. cinereum. Da aber die Resorption der grauen Salbe nicht mit der wünschenswerten Schnelligkeit vor sich geht, verbinde ich die Einreibungen in den ersten zwei bis drei Tagen mit der intramuskulären Einspritzung eines leichtlöslichen Quecksilbersalzes. Als solches habe ich eine $1 \%$ ige Lösung von Hydrargyrum bijodatum in $10 \%$ Kalium jodatumLösung gewählt und gebe hiervon bei Erwachsenen $1 \mathrm{ccm}$ in die Glutäen. Schließlich verwende ich das Quecksilber noch in Form der subkonjunktivalen Einspritzung von $0,3 \mathrm{ccm}$ einer Sublimatlösung 1:2000. Freilich können wir heute wohl nicht mehr daran zweifeln, daß die Quecksilberlösung nicht in den Bulbus gelangt; sie kann also dort nicht antiseptisch wirken. Vielmehr dürfen wir nach den Untersuchungen von Melling er ${ }^{\text {:) }}$ und Wessely2) als sicher annehmen, dab sie, ebenso wie Kochsalzlösungen, lediglich hyperämisierend auf die Gefäße des Ciliarkörpers (und der Iris) wirkt. Trotzdem bevorzuge ich, wenigstens in den ersten Tagen, die Quecksilberinjektionen, da ich mich klinisch überzeugt zu haben glaube, dafi sie energischer wirken, als Kochsalz. Eine Bestätigung dieser klinischen Erfahrung erblicke ich in den Untersuchungen Wesselys, der experimentell feststellte, daß die Beeinflussung des Ciliarkörpers bei subkonjunktivaler Injektion auch isotonischer Lösungen verschiedener Stoffe verschieden groß ist und sich nach der osmotischen Kraft gegenüber der Gefäßwand richtet; besonders grob sei sie bei Sublimatlösungen.

Nächst dieser energischen Quecksilbertherapie lege ich, ebenfalls abweichend von der Ansicht vieler Kollegen, großen Wert auf Innehalten der Bettruhe, bis die Entzündung sich ihrem Ablauf nähert. Ich lasse solche Kranke also meist vier bis sechs Wochen im Bett liegen, und ich habe mich verschiedentlich überzeugt, daß zu frühes Aufstehen mit einem Rezidiv der Entzündung beantwortet wird.

Neben den eben kurz skizzierten, therapeutischen Maßnahmen wurden natürlich die alten, bewährten Medikationen nicht vernachlässigt, also Galvanokaustik in geeigneten frischen, besonders eitrigen Fällen, Atropin, Verband, Schwitzen und nach Schluß der Wunde warme Umschläge. Großes Gewicht lege ich ferner auf schnelle und exakte Wundheilung und suche diese durch sofortige Abtragung etwa vorgefallener Contenta bulbi und konjunktivale Deckung der Wunde, seltener durch Wundnaht, zu erreichen. Hingegen habe ich die besonders von $\mathrm{Haab}^{3}$ ) empfohlene, intraokulare Einführung von

1) Archiv für Augenheilkunde 1894, Bd. 29, S. 238 . - 2) Deutsche medizinische Wochenschrift 1903 , S. 120 . - 3) Bericht aber den neunten internationalen Kongreß für Ophthalmologie zu Utrecht 1899, S. 425.
Jodoform nach einer Reihe von Mißerfolgen ${ }^{1}$ ) wieder aufgegeben, ohne aber damit ein abschließendes Urteil abgeben zu wollen. Für ein solches wäre vor allem nötig, dab die Anhänger dieser Methode ihre Resultate in einer Form veröffentlichen, die einen Vergleich mit meinen Resultaten gestattet.

Wollen wir über den Wert einer Behandlungsmethode bei infizierten Verletzungen ein sicheres Urteil gewinnen, so ist es nötig, alle innerhalb eines gewissen Zeitraums zur Behandlung gekommenen infizierten Verletzungen in die Statistik aufzunehmen. Es genügt durchaus nicht, einzelne glänzende Erfolge zu publizieren; es genügt auch nicht, alle mit dem neuen Mittel behandelten Fälle zu veröffentlichen. Denn die Gefahr liegt zu nahe, daß dann gerade die schwersten Fälle, die doch hoffnungslos erscheinen, mit dem neuen Mittel nicht behandelt werden, sondern nur die leichteren Infektionen. Je nach der Auswahl, die der Autor trifft, hat er es dann in der Hand, seine Statistik günstiger oder weniger günstig zu gestalten. Vergleichbare Resultate, soweit das bei der Verschiedenheit des Krankenmaterials überhaupt möglich ist, erhalten wir nur, wenn wir auf jede Auswahl verzichten und alles in die Statistik aufnehmen. Hierbei würde es bei eingreifenden $\mathrm{Be}$ handlungsmethoden, die nicht in jedem Fall angewandt werden sollen, allerdings ein notwendiges Aequivalent sein, daß ihr Autor auch die mit einfacheren Methoden behandelten leichten Fälle zu seinen Gunsten schreibt. Die Statistik gibt dann ein eindeutiges Bild von den Heilerfolgen des Autors unter Mitverwendung oder hauptsächlicher Verwendung einer bestimmten Heilmethode.

Auf diesen Prinzipien basieren die Tabellen, die ich vor fünf Jahren ${ }^{2}$ ) publiziert habe. A usgeschlossen hatte ich nur die voll ausgebildeten Panophthalmien, welche schon die klassische Trias Lidödem, Chemose und Exophthalmus zeigten; hier hat sich bisher jede Behandlungsmethode machtlos gezeigt.

Die damals berichteten günstigen Erfolge haben eine Anzahl Kollegen zur Nachprüfung meiner Behandlungsmethode ver. anlaßt, und Bentzen ${ }^{3}$ ), Nieden ${ }^{4}$ ) und Lange ${ }^{5}$ ) haben über gïnstige Heilerfolge berichtet. Allgemeinen Eingang in die ophthalmologische Praxis hat die Methode aber bisher nicht gefunden. Der Grund hierfür dürfte einmal darin zu suchen sein, daf es sich um ein recht energisches Verfahren handelt. Einreibungen von 6-9 g Ung. cinereum täglich, die Einverleibung von 150 bis zuweilen über $200 \mathrm{~g}$ im Verlaufe von 9-12 Wochen sind natürlich angreifend für den Patienten. Nach meiner Erfahrung, die sich nunmehr schon auf mehr als 10 Jahre und auf über 150 Patienten erstreckt, kann ich aber versichern, daß die Angegriffenheit durchaus nicht sehr hochgradig ist - vielleicht wirkt hier die Bettruhe günstig - und daß die Kranken sich durchweg schnell erholen. AuBer bald vorübergehenden Quecksilberexanthemen, die mich gewöhnlich veranlaßten, die Inunktion mit dem Mercolintschurz zu vertauschen, und auber leichteren Stomatitiden, die aber nur in selteneren Fällen zwangen, das Mittel für einige Tage auszusetzen, habe ich niemals irgend eine lokale oder allgemeine Störung gesehen. Regelmäbige Eiweißuntersuchungen des Urins, die ich besonders bei Kindern vornehmen lieb, hatten bis auf zwei leichte, schnell vorübergehende Fälle stets ein negatives Resultat.

Das zweite Moment, das sich einer konsequenten Durchfïhrung der Inunktionen entgegenstellt, ist die Furcht vor sympathischer Entzündung. Sehr viele Kollegen haben die Gewohnheit, bei jeder infizierten Bulbuswunde, wenn die Entzündung nicht sehr bald zurückgeht, zu enukleieren, oder wenn nach eingetretener Besserung ein Rückfall der Entzündung eintritt, zu enukleieren oder wenn sich ein gelber Reflex in der Tiefe zeigt, zu enukleieren. Ich habe die feste Ueberzeugung, daß ungezählte Augen unnötigerweise geopfert werden. Ist es doch mir selbst zweimal passiert, daß ich Augen mit guter Sehschärfe gerettet

1) Franck, Weitere Erfahrungen über die Beharidlung der perforierenden infizierten Augapfelverletzungen mit hohen Quecksilbergaben. Dissettation, Greifswald 1904 und Beiträge zur Augenheilkunde 1904, Bd VI, S. 795. - 2) Archiv für Ophthal1904 und Beiträge zur Augenheilkunde 1904, Bd VI, S. 795. - 2) Archiv für Ophthalmologie 1901, Bd. 53, S 1. - 3) Klinische Monatsblätter für Augenheilkunde 1902,
Bd. 40, 2, S. 199. - 4) ibid. 1903, Bd. 4l, 2, S. 82 - 5) Klinische Monatsblätter für Bd. 40, 2, S. $199 .-4$ ) ibid. 1903,
Augenheilkunde 1903 , Bd. $41,2$. 
habe, deren Träger schon, zur Enukleation bereit, auf dem Operationstisch lagen. Unter meinen 157 infizierten Verletzungen habe ich nur zweimal sympathische Entzündung gesehen; beide Male war meine Quecksilbermethode unschuldig.

Im ersten Falle wurde Quecksilber überhaupt nicht angewandt, weil ich bis zum Ausbruch der sehr leichten Cyclitis serosa sympathica zweifelte, ob die Wunde infiziert sei. Erst nachträglich traten auch am ersten Auge zwei Descemetsche Beschläge auf; die Sehschärfe sank auf keinem der beiden Augen jemals unter die Norm; in vier Wochen waren unter Inunktionen beide Augen geheilt. Im zweiten Falle war die Entzündung nicht geheilt, und es sollte zur Sicherstellung des zweiten Auges die Resectio opticociliaris gemacht werden. Infolge einer profusen Blutung mußte jedoch daranf verzichtet werden, und es wurde nur die Neurotomie gemacht, die sich, wie schon öfters, als unzureichend erwies.') 115 Tage nach der Neurotomie trat eine, übrigens in völlige Heilung mit gutem Sehvermögen ausgehende sympathische Entzündung auf. Bei diesem langen Intervall würde die Enukleation bestimmt, wahrscheinlich auch die Resektion den Ausbruch der sympathischen Entzündung verhütet haben.

Da sich unter jenen 157 infizierten Verletzungen sicher eine ganze Reihe sympathiefähiger Bulbi befunden haben, liegt die Annahme nahe, daß das Quecksilber, das ja den ganzen Körper durchdringt, auf die Entzündungserreger nicht nur im verletzten Auge, sondern auch während ihrer Ueberwanderung einen deletären Einfluß ansübt. Hierin würde ein grober Vorzug vor dem Jodoform liegen, das natürlich nur im Bulbus antiseptisch wirken kann. In der Tat ist bei einer viel geringeren Zahl publizierter Fälle nach Jodoformeinführung schon dreimal sympathische Entzündung beobachtet worden.2)

Betrachten wir nunmehr die Heilresultate, welche ich in den letzten zehn Jahren mit den oben geschilderten Behandlungsweisen gehabt habe. Behandelt wurden in dieser Zeit nach Ausschaltung der Fälle, die schon als ausgebildete Panophthalmien in meine Behandlung traten, 157 frische infizierte Verletzungen. Hiervon boten sechs das Bild der Cyclitis serosa; sie wurden sämtlich geheilt; von ihnen soll hier weiter nicht die Rede sein. Das Bild der Uveitis fibrinosa boten 70 , das der Uveitis purulenta 81. Folgende kleine Tabelle zeigt die so gewonnenen Resultate:

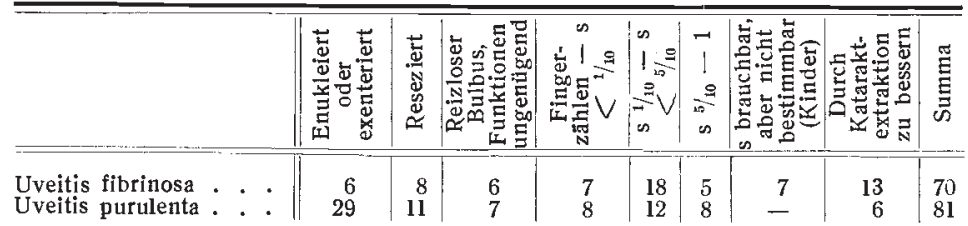

Rechne ich als Verluste alle Fälle, in welchen es nicht gelang, die intraokulare Entzündung zur Heilung zu bringen, und wo es deshalb nötig wurde, den verletzten Bulbus zu enukleieren, zu exenterieren oder zu resezieren, so verzeichne ich bei der Uveitis fibrinosa $80 \%$, bei der Uveitis purulenta $50 \%$ Heilungen. Die letztere Zahl gewinnt erst dadurch ihre volle Bedeutung, dab unter den 81 Fiterungen 59 Glaskörperabscesse waren, also die schwerste Form der Bulbusinfektion, und daß 41 der 81 Augen gleichzeitig einen Fremdkörper, meist ein Eisenstïck, in ihrem Innern beherbergten.

Im ganzen sind von den 41 Augen mit Fremdkörpern 27 erhalten worden, also $66 \%$ gegen $50 \%$ aller eitrigen Uveitiden. Es ist auf den ersten Blick höchst erstaunlich, daß die Fälle mit Fremdkörpern eine bessere Prognose geben, als die einfachen Eiterungen. Doch erklärt es sich zwanglos, wenn man bedenkt, daß die ins Auge gelangenden Splitter fast stets minimal sind, gewöhnlich auch im Moment des Abspringens heiß werden und so nur eine geringe Anzahl, gewiß häufig nicht vollvirulenter Keime ins Bulbusinnere bringen. Zudem ist die mechanische Schädigung bei einem Messer- oder Scheerenstich viel erheblicher, als bei dem Eindringen eines Eisensplitters von meist wenigen Milligrammen Gewicht und bei einer schonend und kunstgerecht ausgeführten Extraktion. Die letztere habe ich bei den weitaus häufigsten Splittern, den Eisensplittern, stets so früh wie irgend möglich gemacht.

1) Wrasidlo, Ein Fall von sympathischer Entzundung nach Neurotomia opticociliaris. Dissertation. Greifswald 1902. - 2) Mayweg, Bericht der Heidelberger ophthalmologischen Gesellschaft 1900, S. 154. - 3) La as, Klinische Monatsb. f Augenheilkunde 1903, Bd I, S. 401 . - 4) v. Grosz, ibid. S. 511. meist innerhalb der ersten drei Stunden nach der Aufnahme des Kranken. Die Splitter, die gewöhnlich im Glaskörper saßen, wurden mit dem Riesenmagneten in die vordere Kammer gezogen und von dort mittels Limbusschnitts leicht entfernt. In zwei Fällen war es nötig, die Extraktion durch einen meridionalen Skleralschnitt vorzunehmen, einmal weil der Eisensplitter sich festgekeilt hatte und dem Zuge des großen Magneten nicht folgen wollte, das andere Mal wegen eines Bleistückchens. Beide Operationen waren erfolgreich, die Bulbi überstanden den Eingriff gut und wurden mit einem mäbigen Grade von Sehvermögen gerettet. Einheilung von Fremdkörpern habe ich zweimal erreicht und zwar bei Steinsplittern; beide Male war der Heilungsverlauf des Glaskörperabscesses ungewöhnlich langwierig und von mehrfachen Entzündungsrezidiven unterbrochen.

Ich habe oben zu den Heilungen auch die Fälle gerechnet, wo das Sehvermögen, meist durch Ablatio, verloren gegangen war. Hierbei leitete mich einmal die Erwägung, daß Heilung im wissenschaftlichen Sinne eben Beseitigung der Entzündung bedeutet und weiter, daß auch die Erhaltung der Form des Augapfels, selbst wenn derselbe blind ist, einen großen $\mathrm{Ge}$ winn, zumal für den Arbeiter, bedeutet. Fragt man aber, in wie viel Fällen es gelang, den Verletzten Sehvermögen zu erhalten, so müssen die obigen Zahlen etwas reduziert werden, und zwar für die U. fibrinosa auf $71 \%$, für die U. purulenta auf $41 \%$. Nur in einem kleinen Teil der Fälle handelt es sich um Rudimente von Sehvermögen; über $\mathrm{s}=1 / 10$ hatten $33 \%$ der U. fibrinosa und $25 \%$ der U. purulenta, und diese Zahlen werden teils durch spontane Aufhellung von Medientrübungen, teils durch Extraktion von Wundstar im Laufe der Zeit noch steigen.

Ueberblicke ich noch einmal die gewonnenen Resultate, so glaube ich sagen zu dürfen, daf dieselben geeignet sind, unsere Anschaungen über die Prognose der infizierten Bulbuswunden wesentlich zu modifizieren. Von 157 solchen Augen, die ich in den letzten zehn Jahren behandelt habe, gelang es mir, $103 \mathrm{zu}$ erhalten, das sind $66 \%$, davon $90=57 \%$ mit größerem oder geringerem Sehvermögen. Sympathische Entzündung, die so gefürchtete Komplikation aller infektiösen Uvealentzündungen, kam nicht ein einziges Mal während oder innerhalb der ersten drei Monate nach Aussetzen der Quecksilbertherapie zur Beobachtung. So lange es im Körper vorhanden ist, scheint es also vorbeugend zu wirken. Man hat jedenfalls viele Wochen Zeit, unbesorgt den Verlauf der Entzündung zu beobachten, und Zeit, in allen Fällen, wo die Entzündung nicht einwandfrei beseitigt ist, operativ den zweiten Bulbus sicherzustellen. Notwendig ist nur, daB von Anfang an hinreichend hohe Quecksilberdosen gegeben werden, und dab die Behandlung hinreichend lange fortgesetzt wird.

Auch die Komplikation von infektiöser Entzün dung mitFremdkörper im Bulbusinnern erfährt durch meine Resultate eine unerwartete Beleuchtung. Weit entfernt, die Prognose zu verschlechtern oder gar zu einer völlig ungünstigen zll machen, hat sich im Gegenteil gezeigt, daß, wenigstens bei Eisensplittern, die Vorhersage sogar günstiger ist. Und in therapeutischer Hinsicht müssen wir schließen, daß möglichst frühzeitige Entfernung des Splitters die richtigste Therapie ist. Hierbei ist die Eröffnung der Skleralkapsel, auch die Erweiterung einer schon vorhandenen Wunde zu vermeiden; der Splitter soll durch den Riesenmagneten in die Vorderkammer gezogen und von dort durch Limbusschnitt entfernt werden. Es gelang zwar, in zwei Fällen Steinsplitter in eitrig infizierten Augen unter Ablauf der Entzündung zur reaktionslosen Einheilung zu bringen; der Heilungsverlauf war aber beide Male sehr langwierig und von mehrfachen Rezidiven unterbrochen, sodah es sich nicht empfiehlt, eine solche zuwartende Therapie auf alle Splitter auszudehnen.

Zum Schluß sei noch darauf hingewiesen, daß die Prognose um so besser ist, je frühzeitiger die energische Behandlung eingeleitet wird. Mit jedem Tage, der nach der Verletzung ungenutzt verstreicht, verschlechtert sich die Prognose. Unter 50 Fällen einer Uveitis fibrinosa, die 
innerhalb der ersten drei Tage nach der Verletzung zur Behandlung kamen, befanden sich nur 8 Verluste $=16 \%$; unter 20 Fällen dagegen, die erst nach dem dritten Tage sich einstellten, waren 6 Verluste $=30 \%$, also das Doppelte der vorigen Zahl. Bei der Uveitis purulenta tritt diese Differenz nicht so deutlich hervor, weil von den Fällen, die erst nach dem dritten Tage zur Behandlung kamen, die schwersten schon in Panophthalmie übergegangen waren, also in meiner Tabelle nicht mehr enthalten sind. Von den noch nicht panophthalmitischen Bulbis kamen 49 bis zum dritten Tage nach der Verletzung in meine Behandlung; hiervon wurden 28 gerettet, 21 gingen verloren $=42 \%$ Verluste. Nach dem dritten Tage kamen 32 Bulbi mit 18 Verlusten $=57 \%$. Unter Zurechnung der Panophthalmien würde obige Zahl auf etwa $80 \%$ Verluste in die Höhe schnellen, während innerhalb der ersten drei Tage nur sehr wenige Panophthalmien zur Behandlung kamen.

Diese Zahlen sprechen deutlich genug. Sie sind für die Herren Kollegen in der Praxis eine dringende Mahnung, jede Verletzung, bei welcher sie Verdacht auf Infektion haben, ohne jeden Zeitverlust in spezialistische Behandlung zu schicken; denn die Therapie erfordert in diesen Fällen eine solche stete Sorgfalt und Beaufsichtigung, dal sie nur in einer Klinik durchgeführt werden kann. Wird uns aber der Kranke früh genug zugeschickt, so können wir versprechen, von drei Fällen zwei geheilt wieder nach Hause zu entlassen. 